\title{
Aftermath Pandemic Challenges for the Cancer Service Provision, The Need for A Fitting Strategy: A Standpoint from the UK
}

\author{
Yirupaiahgari KS Viswanath* \\ Professor of Surgery, Honorary Professor Teesside University, UK
}

*Corresponding author: Yirupaiahgari KS Viswanath, Professor of Surgery, Honorary

Professor Teesside University, Cleveland, UK.

Received Date: June 4, 2021

Published Date: June 17, 2021

\section{Short Communication}

Soon after the 1st lockdown was imposed in the UK on March 27, 2020, all elective activity surgical and endoscopic stopped in James Cook University Hospital, Cleveland County in North England. It was almost a standstill due to fear of the unknown and senior upper GI oncology surgeon thinking how one could not stop cancer-related care, especially extensive major cancer surgeries such as esophagectomy and gastrectomy. Approach with common sense, untold braveness with available guidance and safety kit, surgeries continued despite the risk to the operating surgical team and the patient. The UK health ministry's announcement was candid and stated the cancer care should continue, and one must not deny timely access to the treatment. Furthermore, potential patients' reluctance to attend hospitals, reduced access to primary care, delayed referrals, and a few other factors impacted deferred diagnosis. The estimated loss could amount to 60,000 life-years plus in the next decade in the UK [1].

In contrast to 2019 March to September data, in 2020, 30,000 fewer cancer patients received their first treatment, an unwelcome sign. The UK cancer services recovery plan has developed an action plan to restore the demand to the pre-pandemic level, reduce waiting times and guarantee enough capacity to deliver cancer care [2]. It's now nearly a year on; the UK is on the verge of coming to terms with the end of the pandemic's second wave; however, uncertainty continues with a little more confidence. In the UK, cancer specialist coordination hubs came together to support cancer care at a regional level. Along with this, the timely publication of the National Institute for Health and Care Excellence (NICE) guidance for cancer specialists was invaluable [3]. The advice aimed to maximise the cancer patient safety, best use of NHS resources, and protect the treating medical professionals. Recently published evidence from the UK suggests that many patients acquired Covid while in the hospitals, raising alarms about adherence to recommended protocols such as green, amber, and red patient pathways (traffic light system).

In our hospital setup, we had two patients who acquired Covid who recovered after uneventful surgery. At around the same time, the other two got Covid while waiting for cancer resection, resulting in delayed surgery. Pandemic ensued the medical force to think differently, and last 3 to 4 months, virtual preassessment has become more of a regular event with prehabilitation clinics (PHC). In the latter, virtually surgical patients are engaged, taught, and supervised to enhance the patient well-being and prepare for major cancer surgery. PHC targets four pillars around diet, lifestyle, smoking, alcohol cessation, increased activity. Soon after we thought that dust is settling, another storm took us back to square one, a saga for the last 14 months during this pandemic. Albeit did take measures, and an ongoing vaccination programme has provided the necessary boost to treating professionals, it is still a long way to safeguard our patients and medical force. Less invasive diagnostic interventions such as capsule endoscopy and cytosponge are trialled in the UK to evaluate their routine use in place of invasive endoscopy. A total of 40 trial sites has been identified to recruit 
non-invasive miniature capsule endoscopy, an innovative national approach [4]. The latter part of the second Covid spike, pleasing to note cancer treatment and referral numbers, is returning to usual numbers.

Macmillan cancer support published guidance is a valuable tool to cancer patients and families with friendly illustrations, including video talks from cancer specialists [5]. Likewise, in many other cancers (GI and non-GI), multimodal treatment regimens are used to treat oesophageal and gastric cancer; moreover, performing surgery, especially two or three-stage radical esophagectomy lasting at least two sessions (6-8 hrs) wearing PPE masks or respirators remains challenging. Although, pre-surgery covid screening tests and driven protocols have eased the stress of wearing masks and suits. On top of this, post-surgery management in a green pathway in the critical care area in most hospital setups is not too easy, although there are exceptions. The morbidity and mortality are prohibitively high when a patient develops postoperative pulmonary complications with Covid [6].

In these circumstances, we need to establish ground principles and develop pathways with the proper protocols as preparation in the forthcoming months and years. One has to adopt proactive and reactive strategies with stringent measures to continue providing a quality service for cancer patients. Presurgery and intervention Covid swabs, isolation protocols and weekly twice lateral flow tests for all working staff are a few measures that hold some promise for the safe delivery of cancer care. During the last 14 months of the Covid pandemic, the learned and published evidence should be utilised for this preparation, and one must be mindful of future pandemics. In upper GI cancer surgery and other oncosurgical specialities, one needs to develop a national collaborative consensus with multiple speciality organisations and publish the guidance. The ruling government's support to inject confidence into treating cancer professionals, including onco-surgeons, is indispensable. Virtual meetings, MDT's and clinics have become a norm, while face to face interface has become a luxury. Both patients and professionals have recognised the significance of digital communications for the first time in this millennium. Similar measures are equally applicable to other cancers.

Changing patterns of COVID-19 through mutations (alpha, beta, gamma, delta and more so), although it is not a chameleon mutation, remains a challenge that raises scientists' need to keep ahead of its genomics. Already few of the pharmaceutical industry is preparing booster vaccines that can fight against the new variants. Phase 1 , Covid nasal vaccine trials, shed some light on the end of this pandemic and the new normal. We have to look forward (yet) best of times, forget the worst of times, and follow WHO recommendations. All governments aim to keep Covid-19 near ZERO, which requires collaboration, sacrifice and leadership on all fronts. All hospitals should establish traffic light pathways or equivalent ones with the needed infrastructure to sustain patient safety and uphold quality outcomes for cancer patients. A propensity to hang loose and deviance from recommended protocols should be dispirited.

\section{Acknowledgement}

None.

\section{Conflict of Interest}

No conflict of interest.

\section{References}

1. The Lancet Oncology (2020) UK cancer care threatened by government incompetence. Lancet Oncol 21(11): 1387.

2. (2020) NHS Cancer Programme; COVID-19-Cancer services recovery plan.

3. (2020) COVID-19 rapid guideline: delivery of systemic anticancer treatments. NICE guideline (NG161).

4. (2021) NHS rolls out capsule cameras to test cancer.

5. (2021) Cancer treatment and Coronavirus. Macmillan Cancer Support.

6. COVID Surg Collaborative (2020) Mortality and pulmonary complications in patients undergoing surgery with perioperative SARSCoV-2 infection: an international cohort study. Lancet 396: 27-38. 